\title{
Long-Term Post-Treatment MR and PET/CT Imaging in Prostate Cancer Patients in Complete Remission after Curative Radiotherapy with or without Androgen Deprivation: The Image of Cure
}

Raymond Miralbell ${ }^{1,4}$, Hansjörg Vees ${ }^{1 *}$, Osman Ratib ${ }^{2}$, Michael Wissmeyer ${ }^{2}$, Charles Steiner ${ }^{2}$, Yann Seimbille ${ }^{2}$, Haleem G. Khan ${ }^{3}$ and Fran Buchegger $^{2}$

${ }^{1}$ Department of Radiation Oncology, University Hospital, Geneva, Switzerland

${ }^{2}$ Department of Nuclear Medicine, University Hospital, Geneva, Switzerland

${ }^{3}$ Institute of Radiology Jean Violette, Geneva, Switzerland

${ }^{4}$ Servei de Radio-oncologia, Institut Oncològic Teknon, Barcelona, Spain

\begin{abstract}
Purpose: To investigate the risk of false positive MRI and 18F-Fluorocholine (FCH) or 11Cacetate (AC) PET/ $\mathrm{CT}$ in prostate cancer patients with long term biochemical and clinical remission after curative radiation therapy (RT).

Methods and Materials: Twenty patients underwent MRI studies including T2-weighted, diffusion weighted, dynamic contrast-enhanced, and spectroscopic imaging. Nine of these patients were selected to have $\mathrm{FCH}$ and 10 AC PET/CT studies.

Results: MR spectroscopy suggested positive or equivocal results in $16 / 20$ patients. However, the combined interpretation of all MRI sequences dropped the rate of suspicious or equivocal studies to $8 / 20$. All but one patient underwent also PET/CT and early PET frames showed suspicious or equivocal local images in 15/19 patients (8/10 with $\mathrm{AC}, 7 / 9$ with $\mathrm{FCH}$ ). However, a combined evaluation of early and late PET frames was judged equivocal in only 1 patient.

Conclusions: Multimodal MRI, early single FCH or AC PET frames of the prostate after curative RT may not be accurate enough to differentiate remission from early recurrence. Multiple-phase evaluation of PET images, however, may reduce significantly the risk of reporting equivocal or false positive results.
\end{abstract}

Keywords: Prostate cancer, Radiation therapy, PET/CT, ${ }^{18} \mathrm{~F}$ Fluorocholine, ${ }^{11} \mathrm{C}$-acetate, MRI

\section{Introduction}

Prostate cancer is the most common malignancy and the second leading cause of cancer death in men [1]. Most patients have localized disease at diagnosis and are treated with definitive radiation therapy (RT) or radical prostatectomy [2]. A substantial number of prostate cancer patients treated with external beam RT and developing biochemical recurrence will have local recurrence as the only site of disease [3].

Early locally recurrent prostate cancer cannot be reliably detected with conventional radiologic imaging methods such as CT or transrectal ultrasound (TRUS). Endorectal morphological MRI can provide accurate information concerning local disease at initial presentation $[4,5]$. However, the detection of recurrent cancer with endorectal T2-weighted MRI in the irradiated prostate is limited by treatment related changes that include prostatic shrinkage, diffuse low $\mathrm{T} 2$ signal intensity, creation of aberrant vasculature, and indistinctness of the normal anatomy thus making radiological diagnosis of local recurrent disease difficult $[6,7]$. A higher specificity compared with T2-weighted endorectal MRI has been attributed to multimodal MRI (functional imaging) using spectroscopy, diffusion weighted imaging (DWI), and dynamic contrast-enhanced (DCE) MR [8,9].

Acetate- or choline-based PET/CT are widely used imaging modalities for the detection of recurrent prostate cancer. However, an abnormal tracer uptake after RT may not only be the consequence of a tumor recurrence but also of a non-specific inflammatory benign process.
The purpose of this study was to prospectively investigate the value of multimodal MRI and PET/CT studies with either ${ }^{18} \mathrm{~F}$-Fluorocholine $(\mathrm{FCH})$ or ${ }^{11} \mathrm{C}$-acetate $(\mathrm{AC})$ in patients assumed to be cured after curative radiotherapy for prostate cancer based on a long term $(>5$ years after radiotherapy) low and stable PSA levels and no clinical evidence of recurrence.

\section{Material and Methods}

From December, 2007 to July, 200820 eligible patients with a median age of 71 years (range, 53-82) accepted to participate in this study after ethical committee approval. All patients underwent a multimodal MRI study. Although, 20 patients were planned to undergo a PET/CT study (10 with FCH and 10 with AC) all but one patient accepted to undergo the PET/CT examination.

Patients were selected based on a PSA of $<0.5 \mathrm{ng} / \mathrm{ml}>5$ years after a curative radiotherapy and $>3$ years after completion of the

*Corresponding author: Hansjörg Vees, Department of Radiation Oncology, Geneva University Hospital, CH-1211 Geneva 14, Switzerland, Tel: +41 22 3827090; Fax: +41 22 382711; E-mail: Hansjorg.vees@hcuge.ch

Received May 02, 2011; Accepted December 06, 2011; Published December 08, 2011

Citation: Miralbell R, Vees H, Ratib O, Wissmeyer M, Steiner C, et al. (2011) Long-Term Post-Treatment MR and PET/CT Imaging in Prostate Cancer Patients in Complete Remission after Curative Radiotherapy with or without Androgen Deprivation: The Image of Cure. J Nucl Med Radiat Ther 2:118. doi:10.4172/21559619.1000118

Copyright: (c) 2011 Miralbell R, et al. This is an open-access article distributed under the terms of the Creative Commons Attribution License, which permits unrestricted use, distribution, and reproduction in any medium, provided the original author and source are credited. 
Citation: Miralbell R, Vees H, Ratib O, Wissmeyer M, Steiner C, et al. (2011) Long-Term Post-Treatment MR and PET/CT Imaging in Prostate Cancer Patients in Complete Remission after Curative Radiotherapy with or without Androgen Deprivation: The Image of Cure. J Nucl Med Radiat Ther 2:118. doi:10.4172/2155-9619.1000118

adjuvant androgen deprivation with total testosterone blood levels back to normal. Fourteen patients had been treated with neoadjuvant and concomitant androgen deprivation and curative RT, while six patients had been treated with RT alone. Five patients had received a boost to the prostate with high-dose rate (HDR) brachytherapy. Patient and treatment characteristics are summarized in [Table 1,2] presents detailed information in a patient by patient basis on disease characteristics at diagnosis (PSA, stage, Gleason) and the PSA at imaging and at last follow-up.

\section{MRI acquisition}

The MR images were obtained with a $1.5 \mathrm{~T}$ system by using a combination of an endorectal coil and a pelvic phased array coil. In all patients peristalsis was suppressed by intramuscular injection of 1 mg of Glucagon. First, multisection Turbo SpinEcho images (T2TSE, Repetition time (Rep.T) 7330 ms, Excitation time (ET) 85 ms, NEX 3) were obtained in three orthogonal planes covering the prostate and the seminal vesicles (30 slices with $5 \mathrm{~mm}$ thickness were acquired). Then high resolution TSE images covering the same area were obtained (19 slices, 3mm thickness, Rep.T 4400ms, ET 109ms NEX3). Second, 3D MR spectroscopy (MRS) was performed using a 3D CSI SE protocol. Voxel resolution was $6.7 \times 6.7 \times 6.7 \mathrm{~mm}$. Third, 3D T1 weighted images (Flash 3D) were acquired for DCE MRI after intravenous injection of a paramagnetic gadolinium chelate. The data was evaluated semiquantiatively by calculating an MR signal enhanced time model. Fourth, functional diffusion weighted imaging pulse sequences with the same slice locations as the T2 sequences were performed. The apparent diffusion coefficient (ADC) was calculated from the DWI parametric images. Diffusion weighted MRI can be evaluated by the measure of mean ADC or by visual analysis.

\section{MRI evaluation}

All MRI exams were interpreted by an experienced uro-radiologist. All MRI studies were viewed first blinded for the PET/CT results. Later on a joint meeting with the nuclear medicine specialists took place for an open and joint reading of both MRI and PET/CT studies.

\begin{tabular}{|l|l|l|}
\hline Characteristics & Median (range) & Number (\%) \\
\hline Age & $71(53-82)$ & \\
\hline Stage & & $3(15)$ \\
\hline cT1c & & $6 \quad(30)$ \\
\hline cT2 & & $11(55)$ \\
\hline cT3 & & $20(100)$ \\
\hline cN0 cM0 & & \\
\hline & $13.8(4.1-38.0)$ & \\
\hline PSA (ng/ml) & & $12(60)$ \\
\hline Gleason score & & $6 \quad(30)$ \\
\hline 5-6 & & $2 \quad(10)$ \\
\hline 7 & & $13(41)$ \\
\hline 8-10 & $76.7(74.0-80.4)$ & $5(25)$ \\
\hline Total dose of RT(Gy) & & $14(70)$ \\
\hline Pelvic nodes irradiation & & \\
\hline Brachytherapy boost & & \\
\hline Androgen deprivation & $6.5(5.2-8.9)$ & \\
\hline Years between treatment and imaging & $0.25(<0.04-0.45)$ & \\
\hline PSA at imaging study (ng/ml) & $0.27(<0.04-1.39)$ & \\
\hline PSA at last follow-up (ng/ml) & & \\
\hline
\end{tabular}

Table 1: Clinical characteristics and referral patterns of the patient population $(n=20)$.
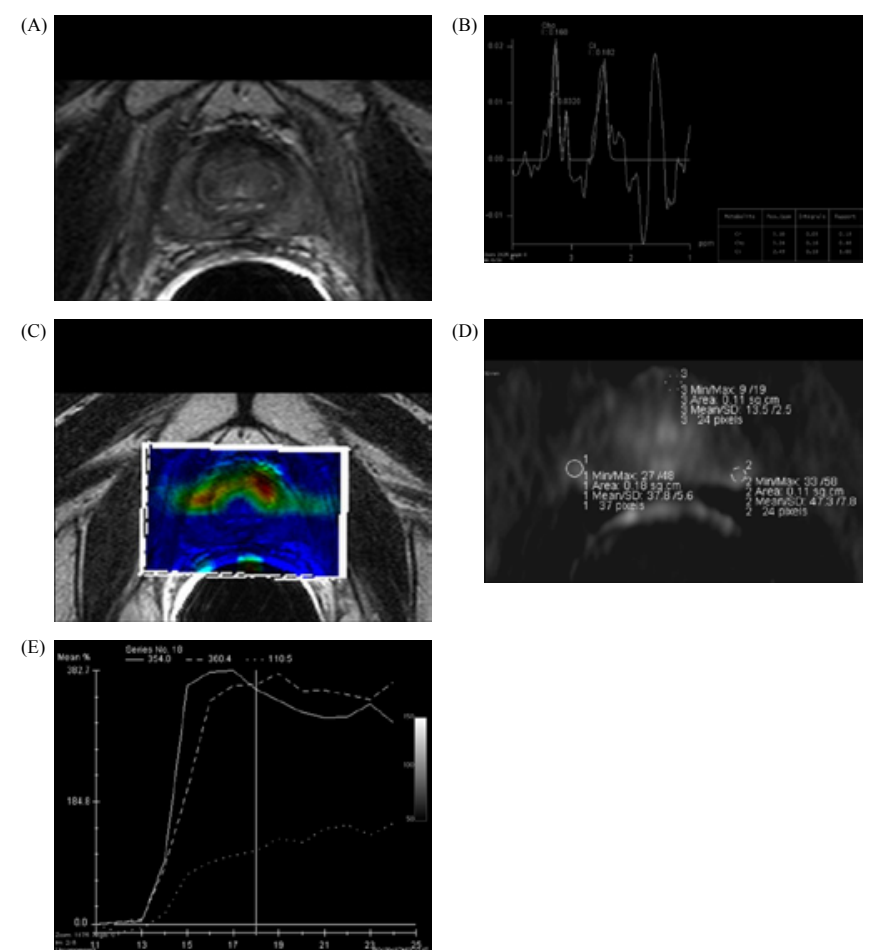

Figure 1: Positive multi-modality MRI study (patient no. 1). A. Negative endorectal T2-weighted MRI. B. MR spectroscopy showing a suspicious lesion in both lobes $(\mathrm{Cho}+\mathrm{Cr} / \mathrm{Ci}$ ratio $=3)$. C-E. Dynamic contrast-enhanced MR study showing suspicious lesions in the peripheral zone of the prostate with a typical cancer wash-out slope.

\section{T2-weighted MRI}

The prostate was divided into base, middle and apex. Each area was subdivided into quadrants so there were 12 regions of interest. The presence of cancer was identified as an area of low signal intensity within the peripheral zone on T2-weighted images or a hypointense or heterogeneous mass effect.

\section{MR spectroscopy}

The MRS imaging was evaluated in the peripheral zone. The peak area ratios of choline plus creatine to citrate $(\mathrm{Cho}+\mathrm{Cr} / \mathrm{Ci})$ for each voxel were calculated. Possible cancer was defined as voxels with a ratio of $\mathrm{Cho}+\mathrm{Cr} / \mathrm{Ci}$ of greater than $2 \mathrm{SD}$ above normal $(>0.75)$. Definite cancer was identified when the ratio of $\mathrm{Cho}+\mathrm{Cr} / \mathrm{Ci}$ was greater than 3 SD above normal $(>0.86)$.

\section{Dynamic contrast-enhanced MRI}

Time-concentration curves were fitted to an exponential model extended with a late washout term. The onset of the exponential curve is defined as the start of enhancement; the exponential constant characterizing the slope and height of the curve is defined as the time to peak. The peak enhancement is the concentration at which the exponential curve becomes level. Washout is defined as the negative slope of the late part of the exponential curve. The start of enhancement was calibrated by using the external iliac artery. Cancer was suspected when the curve exhibited rapid and strong enhancement with a steep initial slope, and a high peak level of enhancement. A significant washout was considered also as highly suspicious for cancer. 
Citation: Miralbell R, Vees H, Ratib O, Wissmeyer M, Steiner C, et al. (2011) Long-Term Post-Treatment MR and PET/CT Imaging in Prostate Cancer Patients in Complete Remission after Curative Radiotherapy with or without Androgen Deprivation: The Image of Cure. J Nucl Med Radiat Ther 2:118. doi:10.4172/2155-9619.1000118

Page 3 of 7

(A)

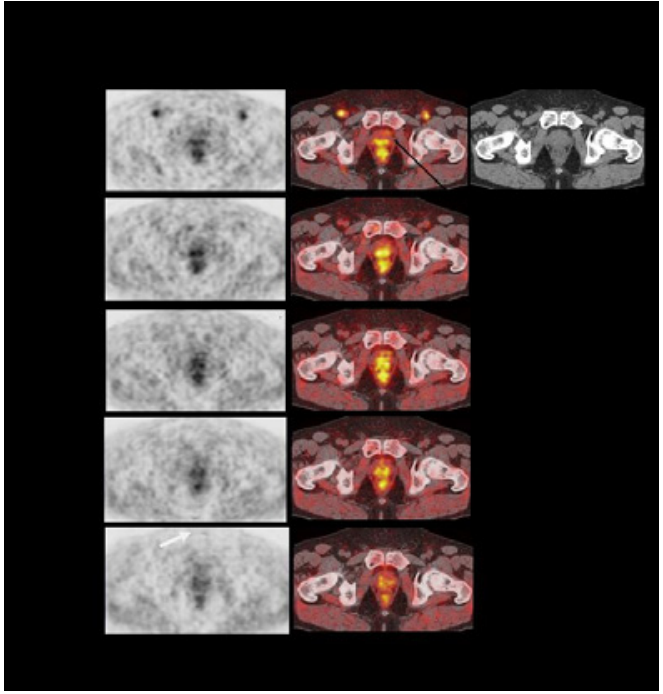

(B)
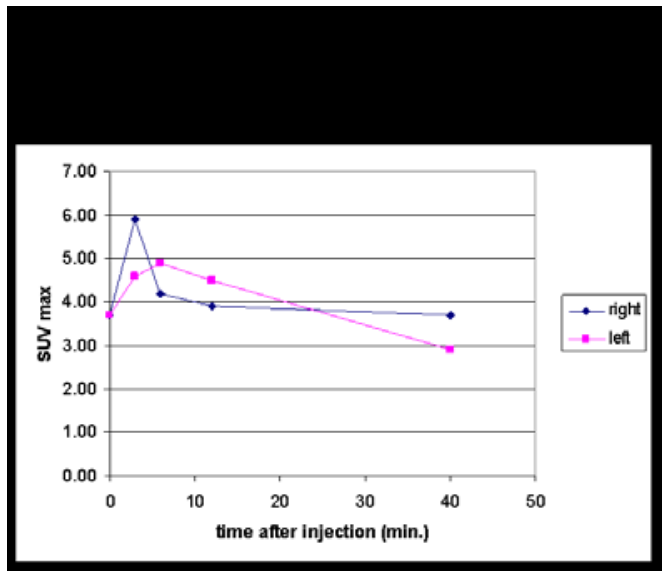

Figure 2: Equivocal three-phase FCH PET/CT display (patient no. 17) showing transverse sections of dynamic frames $(0-3 \mathrm{~min}),(4-6 \mathrm{~min})$ and $(6-$ $9 \mathrm{~min}$ post injection), followed by whole body PET (11-14 min p.i.) and delayed scanning (40 min p.i.), all fused to the initial CT. Sequential disappearance of vascular activity (0-3 min.) and appearance of urinary activity (6-9 min.) is observed (A). Note the differential uptake evolution in the equivocal right lesion (solid line arrow, $\leftarrow$ ) compared with the neg. lesion on the left side. $\mathrm{SUV}_{\text {max }}$ evolution over time (B).

\section{Diffusion weighted MRI}

We performed visual analysis of the DWI images and of the ADC images as well as quantitative ADC measurements.

\section{PET/CT studies}

All patients fasted at least 4 hours before PET study. After bladder voiding, patients underwent an initial CT scan from the mid thigh to the skull performed with arms held above the head and flat breathing.

\section{${ }^{18}$ F-fluorocholine}

FCH (fluorocholinefluoromethyl-dimethyl-2-hydroxyethylammonium) was prepared according to GMP conditions at the Centre of Radiopharmacy, University Hospital of Zürich, Switzerland. Patients received a standard activity of $300 \mathrm{MBq} F C H$. A detailed description of the PET/CT scanning technique has been published elsewhere [10].

\section{${ }^{11} \mathrm{C}$-acetate}

${ }^{11} \mathrm{C}$-acetate was prepared according to GMP requirements at the cyclotron unit of Geneva University Hospital from ${ }^{11} \mathrm{C}$-carbon dioxide produced on an IBA 18/9 cyclotron. The requested radiochemical purity of the patient formulations, as determined by HPLC, was $>95$ $\%$. All patients received a standard activity of $1000 \mathrm{MBq}{ }^{11} \mathrm{C}$-acetate. After the CT scan and five minutes after the injection of the radiotracer the PET study was launched with 7-8 runs of 2 minutes each covering the mid thigh to the skull. A second whole-body PET was started immediately after the first one using 3 minutes per bed position (starting approximately $20 \mathrm{~min}$. after injection) and a final run centred on the prostate was performed immediately after the second scan (approximately $45 \mathrm{~min}$. after injection).

\section{PET/CT imaging analysis and interpretation}

PET/CT interpretation was performed blinded for the MRI results. PET grading was performed according to the typical time course of activity in malignant and benign lesions.

Maximum standard uptake value $\left(\mathrm{SUV}_{\max }\right)$ was calculated within regions of interest (ROIs) drawn over focal hyperactivity using standard formula. For the assessment of $\mathrm{SUV}_{\max }$ time-activity curves $\mathrm{SUV}_{\max }$ values were normalized to the first dynamic frame recorded between 0 and 3 minutes after tracer injection by setting this SUV to $1.0(=100 \%)$. This normalization limits potential bias from partial volume effects in small lesions [11].

All imaging modalities were evaluated on transaxial, coronal and sagittal reconstruction displays. The PET/CT and the MR images were scored as normal, equivocal and abnormal. The functional MRI study was compared with the morphological endorectal T2-weighted study.

\section{Results}

A patient by patient evaluation of all MRI and PET/CT studies is presented in [Table 3].

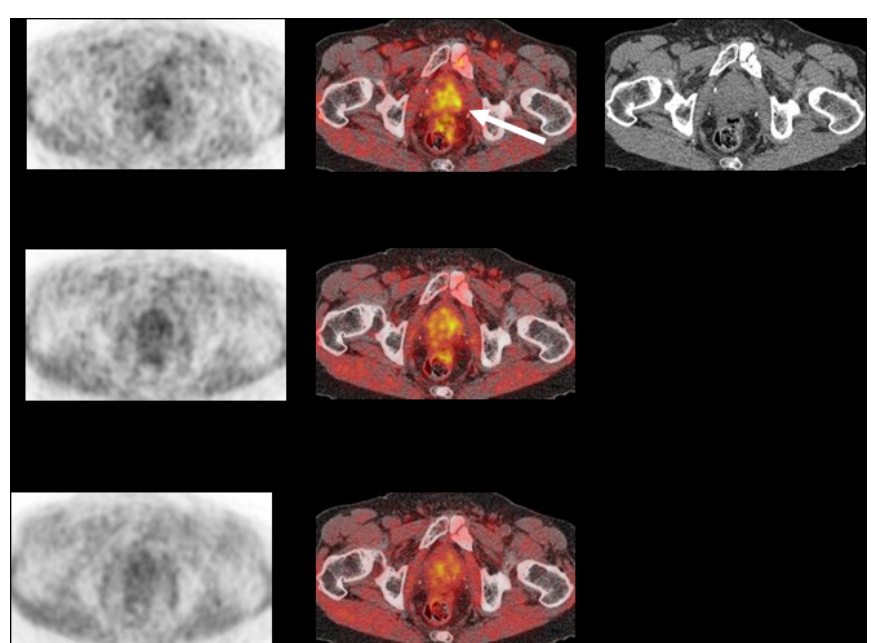

Figure 3: ${ }^{11} \mathrm{C}$-acetate PET/CT (patient no. 6) showing transverse sections of $1^{\text {st }}\left(5 \mathrm{~min}\right.$.), $2^{\text {nd }}\left(20 \mathrm{~min}\right.$.) and a delayed $3^{\text {rd }}$ run $(45 \mathrm{~min}$.) after tracer injection. The $1^{\text {st }}$ and $2^{\text {nd }}$ run showed suspicious and equivocal interpretation of a lesion in the left posterior lobe, respectively (solid line arrow, $\leftarrow$ ). The $3^{\text {rd }}$ run and the global reading were judged as negative. The SUV ${ }_{\max }$ over the 3 runs were $4.4,3.7$ and 2.4 
Citation: Miralbell R, Vees H, Ratib O, Wissmeyer M, Steiner C, et al. (2011) Long-Term Post-Treatment MR and PET/CT Imaging in Prostate Cancer Patients in Complete Remission after Curative Radiotherapy with or without Androgen Deprivation: The Image of Cure. J Nucl Med Radiat Ther 2:118. doi:10.4172/2155-9619.1000118

Page 4 of 7

\begin{tabular}{|c|c|c|c|c|c|c|}
\hline Patient & T stage & Gleason score & PSA at diagnosis (ng/ml) & $\begin{array}{l}\text { PSA at } \\
\text { imaging }(\mathrm{ng} / \mathrm{ml})\end{array}$ & PSA at last follow-up (ng/ml) & $\begin{array}{l}\text { Time between RT and last follow-up } \\
\text { (month) }\end{array}$ \\
\hline 1 & cT3a & $3+3$ & 38.0 & 0.10 & 0.12 & 92 \\
\hline 2 & сT3a & $3+3$ & 20.0 & 0.35 & 0.30 & 72 \\
\hline 3 & cT3a & $3+4$ & 33.5 & $<0.04$ & 0.01 & 85 \\
\hline 4 & cT2 & $3+3$ & 33.7 & 0.09 & $<0.04$ & 91 \\
\hline 5 & cT3a & $5+5$ & 4.9 & 0.45 & 0.37 & 99 \\
\hline 6 & cT3b & $3+3$ & 6.7 & 0.07 & 0.08 & 87 \\
\hline 7 & cT3b & $4+3$ & 7.5 & 0.46 & 0.55 & 87 \\
\hline 8 & cT2b & $4+4$ & 14.0 & 0.08 & 0.03 & 81 \\
\hline 9 & cT2a & $3+3$ & 4.9 & 0.38 & 0.57 & 120 \\
\hline 10 & сT3a & $3+3$ & 13.7 & 0.23 & 1.39 & 108 \\
\hline 11 & cT3a & $3+4$ & 9.3 & 0.23 & 0.26 & 98 \\
\hline 12 & cT1c & $3+3$ & 5.3 & 0.27 & 0.3 & 92 \\
\hline 13 & cT2a & $3+4$ & 13.7 & 0.33 & 0.28 & 91 \\
\hline 14 & cT3a & $4+3$ & 10.4 & $<0.04$ & $<0.04$ & 92 \\
\hline 15 & cT3a & $3+3$ & 15.2 & 0.05 & 0.08 & 80 \\
\hline 16 & cT1c & $3+3$ & 4.1 & 0.05 & 0.46 & 125 \\
\hline 17 & сT3a & $4+4$ & 8.8 & 0.15 & 0.15 & 76 \\
\hline 18 & cT2b & $3+4$ & 14.9 & $<0.04$ & $<0.04$ & 74 \\
\hline 19 & cT1c & $3+3$ & 8.0 & 0.18 & 0.25 & 105 \\
\hline 20 & cT2a & $3+2$ & 9.3 & 0.25 & 0.27 & 128 \\
\hline
\end{tabular}

Table 2: Clinical characteristics.

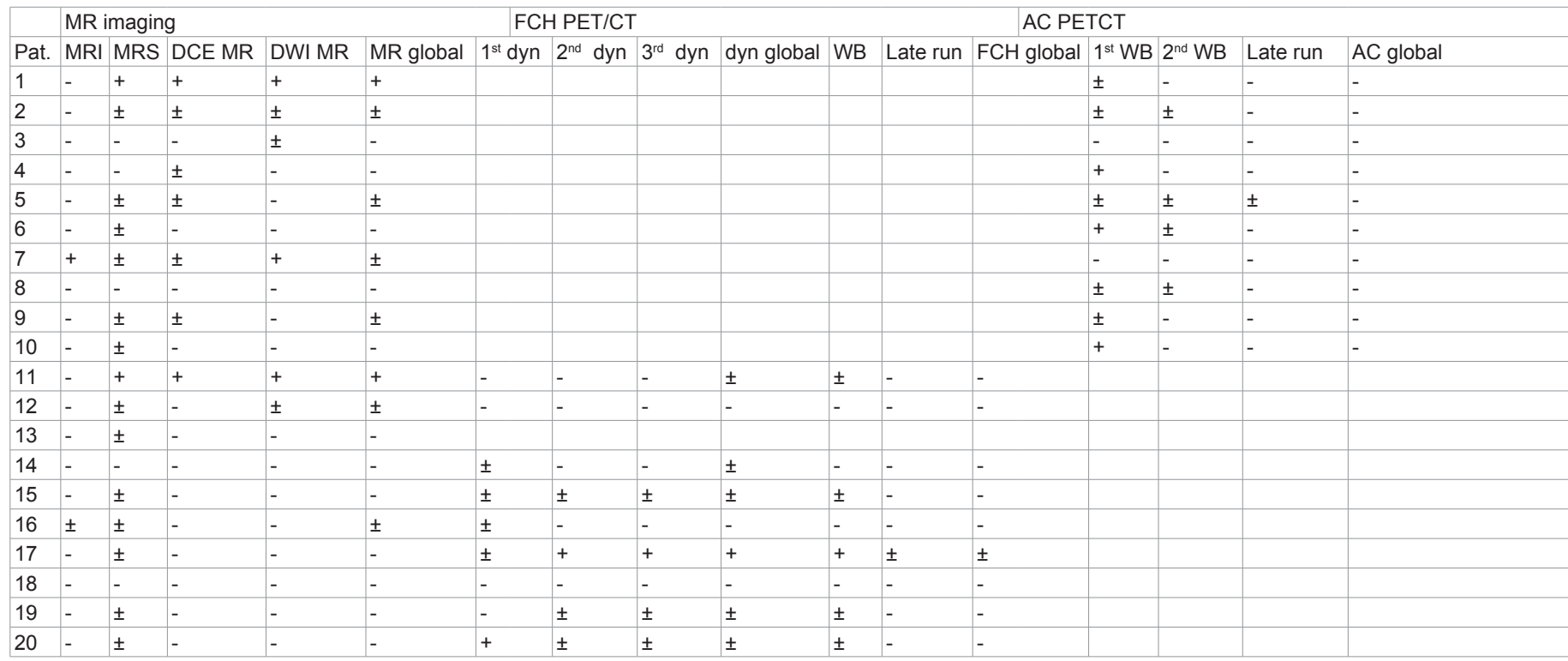

Abbreviations:

"+" = positive

" \pm " = equivocal

"-" = negative

$\mathrm{MRI}=$ contrast enhanced $\mathrm{T} 2$-weighted magnet resonance imaging

MRS = Magnet resonance spectroscopy

DCE $=$ dynamic contrast enhanced

$\mathrm{DWI}=$ diffusion-weighted imaging

$\mathrm{FCH}={ }^{18} \mathrm{~F}$-Fluorocholine

dyn = dynamic PET scan

$\mathrm{AC}={ }^{11} \mathrm{C}$-acetate

WB $=$ whole-body PET scan

A combined evaluation of prostate and seminal vesicle were performed in all patients

Table 3: Summery of all MRI and PET/CT studies

\section{MRI evaluation}

The quality of MRI was rated as excellent in 18 patients and intermediate in 2 . The median prostate volume measured on MRI images was $21 \mathrm{cc}$ (range $12-25)$. The contrast enhanced T1-weighted images showed only 1 suspicious and 1 equivocal lesion while in the remaining 18 patients images were evaluated as negative. The patient with the suspicious result showed a pronounced poorly defined hypointense focus at the left base. There was however, no distinct mass 
effect. The evaluation of the MRS revealed suspicious, equivocal and negative results in 2,13 , and 5 patients, respectively. DCE MRI found suspicious lesions in 2 patients and equivocal lesions in 5 patients. The other 13 patients were estimated as negative. The 2 patients with suspicious DCE MRI revealed abnormal images in the peripheral zone with typical cancer washout slopes. An example of a suspicious DCE $\mathrm{MR}$ is shown in figure 1 . The 5 patients with equivocal results showed less clear curve progressions and/or localisation in the transitional zone. The DWI MRs were judged as negative in 14 patients while suspicious and equivocal lesions were found in 3 and 3 patients, respectively. The 3 suspicious DWI MRs showed small hyposignal regions of 3 to $5 \mathrm{~mm}$ and a reduced ADC in regard to the surrounding prostate tissue. The combined interpretation of all MR sequences still showed suspicious or equivocal results in $10 \%$ and $30 \%$ studies, respectively. In only $60 \%$ of the patients the combined evaluation was clearly negative. There was no evidence of regional or distant metastasis in the pelvis.

\section{PET evaluation}

${ }^{18}$ F-fluorocholine PET/CT: The FCH PET studies were evaluated separately for early, whole body and late PET frames. The evaluation of the whole body PET/CT showed no evidence of regional or distant metastasis. The analysis of the early dynamic PET frames (between 0 and $9 \mathrm{~min}$. after tracer injection) showed suspicious, equivocal and negative images in 1, 5 and 3 patients, respectively. Frames at 11-14 min. after injection were suspicious in 1 patient and equivocal in 4 . Late PET images (40 min. after tracer injection) were equivocal or negative in 2 and 7 patients, respectively. The combined evaluation of all FCH PET frames resulted in 8 negative and 1 equivocal PET [Figure 2].

${ }^{11} \mathrm{C}$-acetate PET/CT: AC PET/CT included two whole body PET frames ( 5 and $20 \mathrm{~min}$. after tracer injection) followed by a late run centred on the prostate ( $45 \mathrm{~min}$. after tracer injection). The evaluation of the whole body PET/CTs showed no evidence of regional or distant metastasis. The analysis of the first run showed suspicious, equivocal and negative images in 3,5 and 2 patients, respectively. The second run did not show suspicious lesions but equivocal images in 3 patients. The remaining 7 studies were judged to be negative. The late run showed negative images in 9 patients, while only 1 patient presented 1 equivocal lesion in the right base of the prostate. All AC PET were interpreted as negative in the combined evaluation and an example is shown in [Figure 3].

A global evaluation of all PET/CT and MRI studies was finally undertaken [Table 3]. All patients continued to be seen on regular follow-up visits after the study with sequential PSA determinations as shown in [Table 2]. The median follow-up time after imaging was 15.7 months (range: 6-21) and 7.9 years (range, 6.0-10.7) after the end of radiotherapy. Although, no biochemical failure was observed, three patients (no 7, 9 and 10) presented with a PSA $>0.5 \mathrm{ng} / \mathrm{ml}$ (i.e. 0.55 , 0.57 , and $1.39 \mathrm{ng} / \mathrm{ml}$, respectively) at last follow-up. For these three patients MRI and PET/CT studies were judged to be equivocal and negative, respectively.

\section{Discussion}

The present study aimed to underscore the potential pitfalls of MRI and PET/CT when used to assess the local disease status of patients after curative radiotherapy with or without androgen deprivation. Different authors have illustrated the limited value of T2-weighted MRI in recurrent prostate cancer after RT $[6,7,12]$. Indeed, RT may cause a reduction in T2-weighted signal intensity secondary to glandular atrophy and fibrosis and low sensitivity and specificity; especially for tumor foci $<1 \mathrm{~cm}$ [13]. Radiation induced anatomic changes in the prostate like reactive stromal fibrosis and ischemia may be detected as a diffuse low T2 signal intensity on MRI, thus limiting its use in the follow-up of prostate cancer after RT [6,7]. Nevertheless, endorectal T2-weighted MRI in our study showed homogeneous prostate tissue without any suspected tumor focus in $90 \%$ of patients.

The value of MRS in recurrent prostate cancer after RT is controversial $[14,15]$. Spectroscopy usually detects prostate cancer based on the ratio between a high choline + creatinine signal (tumor) and a reduced citrate signal (normal tissue). After RT, however, the ratio $\mathrm{Cho}+\mathrm{Cr} / \mathrm{Ci}$ is of limited use due to citrate and choline signals that are often undetectable. MRI studies before salvage prostatectomy have shown sensitivity and specificity rates of $36-76 \%$ and $65-81 \%$, respectively $[14,16]$; thus in good agreement with our findings (i.e., equivocal or false positive images in $75 \%$ of cases).

DCE MRI is deemed to image tumor angiogenesis [17]. Prostate cancer on DCE MRI can be shown by an enhancement curve with a fast wash-in peak and a wash-out slope shallower than the respective curves for normal prostate tissue [18]. Radiation induced fibrosis, however, may be less enhanced and may also show a shallower wash-out slope compared to normal prostate tissue. In our series, we observed suspicious DCE MR studies in two patients. In 5 additional patients DCE MR studies were evaluated as equivocal due to unclear curve slopes.

DWI is deemed to differentiate malignant lesions from benign hyperplasia in the prostate optimizing the diagnostic performance of T2-weighted MRI [19]. The sensitivity of the ADC in prostate cancer is optimal in the peripheral zone but rather poor in the prostate base [20]. Lower ADC values are consistent with restricted water content in the extracellular space secondary to the dense cellularity of malignant tissue [21]. However, these differences may disappear after RT as ADC values may increase in the tumor region due to the radiation induced inflammatory swelling of tumour cells and decrease in the prostatic non-tumoral tissue due to stromal fibrosis possibly explaining the false positives or equivocal DWI studies in our series [22,23].

One of the advantages of PET/CT imaging in comparison to MRI is that it is a whole-body imaging modality with the ability to provide simultaneous information on local recurrence and distant metastasis. A drawback of PET imaging, however, is a limited spatial resolution of $4-8 \mathrm{~mm}$. Metabolic abnormalities on PET studies can also be induced by a latent chronic prostatitis in those patients with relatively low PSA values at the moment of the suspected relapse those that one would be tempted to salvage with unnecessary local retreatment and/or androgen deprivation $[24,25]$.

As shown in the present study, timing of AC PET imaging in the irradiated prostate is of major importance in order to prevent against false positive studies. Kato et al. showed that in comparison with normal or hyperplasic prostate tissue, primary prostate cancer has a higher early-to-late SUV activity ratio for AC [26]. The impact of early and late runs in recurrent prostate cancer has been pointed out in another study of our group [27]. The imaging protocol was extended by a late run $45 \mathrm{~min}$. after $\mathrm{AC}$ injection showing an optimized power to differentiate benign hyperactivity in early imaging from recurrent cancer. Schiepers et al. reported that the acetate kinetics of prostate cancer can be adequately described by a 3-compartement model and that early and late SUV are significantly different in primary and recurrent prostate cancer [28]. In the present study we have been able to show that limiting the study to the first PET frame 5 min. after tracer 
Citation: Miralbell R, Vees H, Ratib O, Wissmeyer M, Steiner C, et al. (2011) Long-Term Post-Treatment MR and PET/CT Imaging in Prostate Cancer Patients in Complete Remission after Curative Radiotherapy with or without Androgen Deprivation: The Image of Cure. J Nucl Med Radiat Ther 2:118. doi:10.4172/2155-9619.1000118

injection may be misinterpreted as tumor recurrence in up to $80 \%$ of patients. The inclusion of a second whole body run and a late run 45 min. after tracer injection helped to exclude suspicious or equivocal images.

One may consider a drawback the fact that rebiopsies of the prostate were not planned in our study, thus being unable to ascertain for a pathologic remission of the local tumor. However, our patients were selected based on a high likelihood of being biochemically disease-free. Indeed, Yock et al observed in an assessment of a group of 328 men disease-free more than five years after external beam RT, that for those patients with a PSA $<0.5 \mathrm{ng} / \mathrm{ml}$ at 5 year, the likelihood of persistent disease-free 10 year after treatment was $92 \%$ ( 7 failures out of 122 patients at risk) [29]. Davis et al, also observed in a similar group of patients treated with external beam RT that if a PSA nadir of $<0.5$ $\mathrm{ng} / \mathrm{ml}$ was maintained 5 years after therapy subsequent failure was rare with one patient among 26 patients at risk failing after 133 months (i.e., 91\%, 10-year disease-free survival) [30]. Only one out of the 19 patients undergoing both MRI and PET/CT studies presented with equivocal results. As shown in tables 2 and 3, the equivocal FCH PET/CT study corresponded to patient nb17, who presented with a stable PSA of $0.15 \mathrm{ng} / \mathrm{ml}$ at the time of the study and 76 months after treatment completion at last follow-up. The patient with the highest PSA at last follow-up (1.39 ng/ml, 108 months after treatment completion) was patient $\mathrm{nb} 10$ who presented with a PSA of $0.23 \mathrm{ng} / \mathrm{ml}$ at the time of recruitment with subsequent negative MRI and AC PET/CT studies [Tables 2 and 3].

Improvements in MR technology will allow an increase in signal to noise ratio. This may decrease discrepancies in voxel size with a more precise voxel to voxel analysis increasing the ability to detect small tumors or recurrences. A new and promising development in imaging technology is a hybrid PET/MR device which may improve lesion characterization [31]. This approach may succeed in enhancing both sensitivity and specificity.

\section{Conclusion}

With the present study we have shown that multimodal endorectal MRI or early single FCH or AC PET frames of the prostate after curative RT may not be reliable enough to differentiate remission from early local recurrence. Multiple-phase evaluation of PET images, however, may reduce significantly the risk of reporting equivocal or false positive results and are to be recommended in patients undergoing PET/CT studies for failure assessment especially those with an early biochemical failure with relatively low PSA values at relapse.

\section{References}

1. Jemal A, Siegel R, Ward E, Hao Y, Xu J, et al. (2009) Cancer statistics, 2009. CA Cancer J Clin 59: 225-249.

2. Jani AB, Hellman S (2003) Early prostate cancer: clinical decision-making. Lancet 361: 1045-1053.

3. Ward JF, Moul JW (2005) Biochemical recurrence after definitive prostate cancer therapy Part I: defining and localizing biochemical recurrence of prostate cancer. Curr Opin Urol 15: 181-186.

4. Testa C, Schiavina R, Lodi R, Salizzoni E, Corti B, et al. (2007) Prostate cancer: sextant localization with MR imaging, MR spectroscopy, and 11C-choline PET/ CT. Radiology 244: 797-806

5. Wang L, Hricak H, Kattan MW, Chen HN, Scardino PT, et al. (2006) Prediction of organ-confined prostate cancer: incremental value of MR imaging and MR spectroscopic imaging to staging nomograms. Radiology 238: 597-603.

6. Coakley FV, Hricak H, Wefer AE, Speight JL, Kurhanewicz J, et al. (2001) Brachytherapy for prostate cancer: endorectal MR imaging of local treatmentrelated changes. Radiology 219: 817-821.
7. Stone HB, Coleman CN, Anscher MS, McBride WH (2003) Effects of radiation on normal tissue: consequences and mechanisms. Lancet Oncol 4: 529-536.

8. Haider MA, Chung P, Sweet J, Toi A, Jhaveri K, et al. (2008) Dynamic contrastenhanced magnetic resonance imaging for localization of recurrent prostate cancer after external beam radiotherapy. Int J Radiat Oncol Biol Phys 70: 425 430

9. Reinsberg SA, Payne GS, Riches SF, Ashley S, Brewster JM, et al. Combined use of diffusion-weighted MRI and $1 \mathrm{H}$ MR spectroscopy to increase accuracy in prostate cancer detection. AJR Am J Roentgenol 188: 91-98.

10. Steiner Ch, Vees H, Zaidi H, Wissmeyer M, Berrebi O, et al. (2009) Three-phase (18)F-fluorocholine PET/CT in the evaluation of prostate cancer recurrence. Nuklearmedizin 48: 1-9.

11. Basu S, Zaidi H, Houseni M, Bural G, Udupa J, et al. (2007) Novel quantitative techniques for assessing regional and global function and structure based on modern imaging modalities: implications for normal variation, aging and diseased states. Semin Nucl Med 37: 223-239.

12. Fuchsjäger M, Akin O, Shukla-Dave A, Pucar D, Hricak H (2009) The role of $\mathrm{MRI}$ and MRSI in diagnosis, treatment selection, and post-treatment follow-up for prostate cancer.Clin Adv Hematol Oncol 7: 193-202.

13. Nakashima J, Tanimoto A, Imai $Y$, Mukai M, Horiguchi $Y$, et al. (2004) Endorectal MRI for prediction of tumor site, tumor size, and local extension of prostate cancer. Urology 64: 101-105

14. Pucar D, Shukla-Dave A, Hricak H, Moskowitz CS, Kuroiwa K, et al. (2005) Prostate cancer: correlation of MR imaging and MR spectroscopy with pathologic findings after radiation therapy-initial experience. Radiology 236 545-553.

15. Pickett B, Kurhanewicz J, Coakley F, Shinohara K, Fein B, et al (2004) Use of $\mathrm{MRI}$ and spectroscopy in evaluation of external beam radiotherapy for prostate cancer. Int J Radiat Oncol Biol Phys 60: 1047-1055.

16. Sala E, Eberhardt SC, Akin O, Moskowitz CS, Onyebuchi CN, et al. (2006) Endorectal MR imaging before salvage prostatectomy: tumor localization and staging. Radiology 238: 176-183.

17. Padhani AR (2002) Dynamic contrast-enhanced MRI in clinical oncology: current status and future directions. J Magn Reson Imaging 16:407-422.

18. Engelbrecht MR, Huisman HJ, Laheij RJ, Jager GJ, van Leenders GJ, et al. (2003) Discrimination of prostate cancer from normal peripheral zone and central gland tissue by using dynamic contrast-enhanced MR imaging Radiology 229:248-254.

19. Xu J, Humphrey PA, Kibel AS, Snyder AZ, Narra VR, et al. (2009) Magnetic resonance diffusion characteristics of histologically defined prostate cancer in humans. Magn Reson Med 61:842-850.

20. Kim JH, Kim JK, Park BW, Kim N, Cho KS (2008) Apparent diffusion coefficient prostate cancer versus noncancerous tissue according to anatomical region. J Magn Reson Imaging 28:1173-1179.

21. Nakayama T, Yoshimitsu K, Irie H, Aibe H, Tajima T, et al. (2004) Usefulness of the calculated apparent diffusion coefficient value in the differential diagnosis of retroperitoneal masses. J Magn Reson Imaging 20:735-742.

22. Petraki CD, Sfikas CP (2007) Histopathological changes induced by therapies in the benign prostate and prostate adenocarcinoma. Histol Histopathol 22 107-118.

23. Takayama Y, Kishimoto R, Hanaoka S, Nonaka H, Kandatsu S, et al. (2008) $A D C$ value and diffusion tensor imaging of prostate cancer: changes in carbonion radiotherapy. J Magn Reson Imaging 27:1331-1335.

24. Shukla-Dave A, Hricak H, Eberhardt SC, Olgac S, Muruganandham M, et al. (2004) Chronic prostatitis: MR imaging and 1H MR spectroscopic imaging findings--initial observations. Radiology 231:717-724.

25. Reske SN, Blumstein NM, Glatting G (2006) [Advancement of PET and PET/ CT in prostate carcinoma]. Urologe A 45: 707-710, 712-714.

26. Kato T, Tsukamoto E, Kuge Y, Takei T, Shiga T, et al. (2002) Accumulation of [11C] acetate in normal prostate and benign prostatic hyperplasia: comparison with prostate cancer. Eur. J. Nucl. Med. Mol. Imaging 29: 1492-1495.

27. Albrecht S, Buchegger F, Soloviev D, Zaidi H, Vees H, et al. (2007) (11) $\mathrm{C}$-acetate PET in the early evaluation of prostate cancer recurrence. Eur. J. Nucl. Med. Mol. Imaging 34: 185-196. 
Citation: Miralbell R, Vees H, Ratib O, Wissmeyer M, Steiner C, et al. (2011) Long-Term Post-Treatment MR and PET/CT Imaging in Prostate Cancer Patients in Complete Remission after Curative Radiotherapy with or without Androgen Deprivation: The Image of Cure. J Nucl Med Radiat Ther 2:118. doi:10.4172/2155-9619.1000118

28. Schiepers C, Hoh CK, Nuyts J, Seltzer M, Wu C, et al. (2008) 1-11C-acetate kinetics of prostate cancer. J. Nucl. Med 49: 206-215

29. Yock TI, Zietman AL, Shipley WU, Thakral HK, Coen JJ (2002) Long-term durability of PSA failure-free survival after radiotherapy for localized prostate cancer. Int J Radiat Oncol Biol Phys 54: 420-426.
30. Davis JW, Kolm P, Wright GL Jr, Kuban D, El-Mahdi A, et al. (1999) The durability of external beam radiation therapy for prostate cancer: can it be identified? J Urol 162:758-761.

31. Judenhofer MS, Wehrl HF, Newport DF, Catana C, Siegel SB, et al. (2008) Simultaneous PET-MRI: a new approach for functional and morphological imaging. Nat Med 14:459-465. 